\begin{tabular}{|c|l|}
\hline Title & Computational visualization of unsteady flow around vehicles using high performance computing \\
\hline Author(s) & $\begin{array}{l}\text { Tsubokura, Makoto; Kobay ashi, Toshio; Nakashima, Takuji; Nouzawa, Takahide; Nakamura, Takaki; Zhang, Huilai; } \\
\text { Onishi, Keiji; Oshima, Nobuyuki }\end{array}$ \\
\hline Citation & $\begin{array}{l}\text { Computers \& Fluids, 38(5), 981-990 } \\
\text { https://doi.org/10.1016/.Compfluid.2008.01.020 }\end{array}$ \\
\hline Issue Date & 2009-05 \\
\hline Doc URL & http://hdl.handle.net/2115/49859 \\
\hline Type & article (author version) \\
\hline File Information & CaF385_981-990.pdf \\
\hline
\end{tabular}

Instructions for use 
Computational Visualization of Unsteady Flow Around Vehicles Using High Performance Computing

\section{Makoto TSUBOKURA}

Division of Mechanical and Space Engineering

Graduate School of Engineering

Hokkaido University

N13, W8, Kita-ku, Sapporo-shi, Hokkaido 060-8628, Japan

Tel: (+81)-11-706-6723

Fax: (+81)-11-706-6723

mtsubo@eng.hokudai.ac.jp

20 pages

15 figures

No tables

Microsoft Office Word 2003 


\title{
Computational Visualization of Unsteady Flow Around Vehicles Using High Performance Computing
}

\author{
Makoto Tsubokura ${ }^{1}$ \\ Toshio Kobayashi $^{2}$ \\ Takuji Nakashima ${ }^{3}$ \\ Takahide Nouzawa ${ }^{4}$ \\ Takaki Nakamura $^{4}$ \\ Huilai Zhang $^{5}$ \\ Keiji Onishi ${ }^{5}$ \\ Nobuyuki Oshima $^{1}$
}

1 Division of Mechanical and Space Engineering, Hokkaido University - Graduate School of Engineering, Japan

2 Japan Automobile Research Institute and University of Tokyo, Japan

3 Department of Social and Environmental Engineering, Hiroshima University - Graduate School of Engineering, Japan

4 Mazda Motor Corporation, Japan

5 Advance Soft, Japan 


\section{ABSTRACT}

One of the largest-scale unstructured Large Eddy Simulation (LES) of flow around a full-scale road vehicle is conducted on the Earth Simulator in Japan. The main objective of our study is to look into the validity of LES for the assessment of vehicle aerodynamics, especially in the context of its possibility for unsteady or transient aerodynamic forces. Firstly, the aerodynamic LES proposed is quantitatively validated on the ASMO simplified model by comparing the mean pressure distributions on the vehicle surface with those obtained by a conventional Reynolds-Averaged Navier-Stokes simulation (RANS) or a wind tunnel measurement. Then, the method is applied to the full-scale vehicle with complicated geometry to qualitatively investigate the capability of capturing organized flow structures around the vehicle. Finally, unsteady aerodynamic forces acting on the vehicle in transient yawing-angle change are estimated and relationship between the flow structures and the transient aerodynamic forces is mentioned. As a result, it is demonstrated that LES will be a powerful tool for the vehicle aerodynamic assessment in the foreseeable future, because it can provide precious aerodynamic data which conventional wind tunnel tests or RANS simulations are difficult to provide.

Keywords: Computational Fluid Dynamics (CFD), High Performance Computing (HPC), Vehicle Aerodynamics, Turbulent Flow, Eddy Structures, Large Eddy Simulation (LES) 


\section{INTRODUCTION}

Computational Fluid Dynamics (CFD) is going to be a powerful tool for the vehicle aerodynamics because it can provide enormous amount of information as well as high economical efficiency. However, the Reynolds-Averaged Navier-Stokes (RANS) model, which is commonly used for vehicle aerodynamics, has two fundamental problems: its strong dependence on turbulence models adopted, and its difficulty of capturing the unsteady flow characteristics. Thus, RANS plays only a supplementary role of a wind tunnel test at the moment.

Recently, greater attention is paid to unsteady aerodynamic forces (e.g., Cogotti [1] and Mayer et al.[2]) generated from sudden steering action, overtaking, or cross wind condition. The most conventional method to assess the stability in a cross wind condition at the moment is based on the quasi-steady method [3] in which "aerodynamic forces caused by wind gusts are supposed to be predicted by assuming the flow to behave in a quasi-steady way." Thus, the mean aerodynamic forces such as the side force or the yawing moment are measured in a wind tunnel facility at various yawing angle in a stationary state. However, limitation of the quasi-steady method has also been pointed out [4][5] in a strong cross wind condition with sudden change of wind velocity. In addition, it is argued by Coleman and Baker [6] that the gust of a natural wind itself alters the flow characteristics around a vehicle. The unsteady or transient aerodynamic forces acting on a full scale vehicle are extremely difficult to measure in a wind tunnel. It goes without saying that RANS is not appropriate for that purpose. Accordingly alternative method to the conventional manners is strongly desired. LES will be an encouraging solution to the problem because it can reproduce unsteady turbulence characteristics with high accuracy, but in turn it requires excessively large computational resources. Consequently, only few attempts have been made so far to apply LES to the assessment of vehicle aerodynamics.

To conquer the problem of LES in practical use for vehicle aerodynamics, as well as to foresee the computer-aided aerodynamic design in the near future, the unstructured-grid LES code "FrontFlow/red (FFR)" based on the unstructured Finite Volume (FV) method was intensively optimized for the execution on the Earth Simulator (ES). The software was developed by us at the University of Tokyo under the national project "Revolutionary Simulation Software (RSS)". Finally, more than 95\% and 99\% of high vectorization and parallelization, respectively, were achieved, which made it possible to conduct one of the world's largest unstructured LES simulations of flows around vehicles.

In this study, we first apply our High Performance Computing (HPC) LES to the ASMO simplified model and compare the results with those obtained by RANS to show the fundamental validity of our method. Then, we apply the method to a full scale commercial sedan to discuss how our HPC-LES can properly reproduce three dimensional unsteady vortex structures around the vehicle. Visualized flow structures obtained by the LES are qualitatively compared with those obtained by a wind tunnel measurement. Finally, relationships between the flow structures and unsteady aerodynamic forces are mentioned through the flow simulation around the vehicle in dynamic yawing angle change.

\section{NUMERICAL METHODS}

\section{Analysis Model and Computational Domain}

The analysis model is a full-scale production vehicle (Mazda Atenza), which involves the engine room and complicated underbody shape. The vehicle length $(\mathrm{L})$, width $(\mathrm{W})$, and height $(\mathrm{H})$ are about $4.7 \mathrm{~m}, 2.0$ $\mathrm{m}$, and $1.6 \mathrm{~m}$, respectively. The size of the computational domain is $13 \mathrm{~L}, 10 \mathrm{~L}$, and $5 \mathrm{~L}$ for streamwise, spanwise, and normal-wall directions, respectively, as illustrated in Fig. 1. To avoid the possible blockage effect, the cross-sectional size is set to be relatively larger than the wind tunnel facility where 
corresponding experiments are conducted.
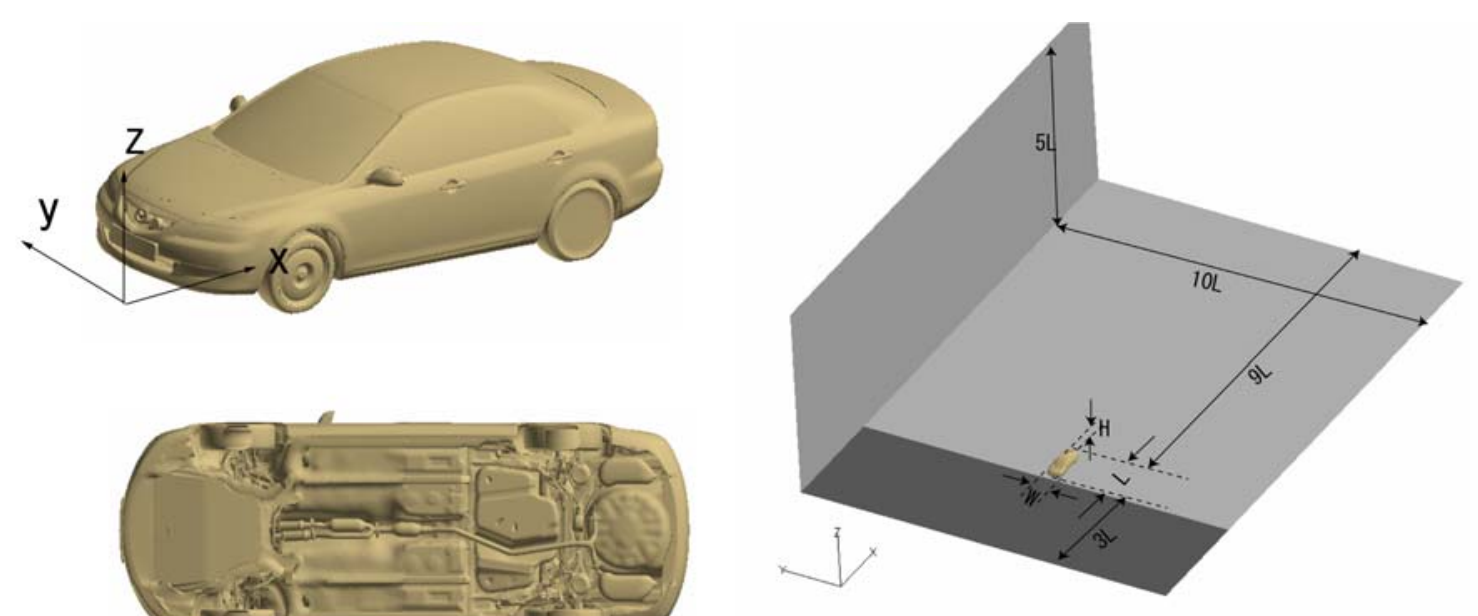

Fig. 1. The analysis model (left) and the computational domain (right).

\section{Governing Equations}

The governing equations adopted in this study are the spatially filtered continuity and Navier-Stokes equations as indicated below:

$$
\begin{gathered}
\frac{\partial \bar{u}_{i}}{\partial x_{i}}=0 \\
\frac{\partial \bar{u}_{i}}{\partial t}+\frac{\partial}{\partial x_{j}} \bar{u}_{i} \bar{u}_{j}=-\frac{\partial \bar{P}}{\partial x_{i}}+2 \frac{\partial}{\partial x_{j}}\left(v+v_{S G S}\right) \bar{S}_{i j} \\
\bar{S}_{i j}=\frac{1}{2}\left(\frac{\partial \bar{u}_{j}}{\partial x_{i}}+\frac{\partial \bar{u}_{i}}{\partial x_{j}}\right) \\
\bar{P}=\bar{p} / \rho+\left(\overline{u_{i} u_{i}}-\bar{u}_{i} \bar{u}_{i}\right) / 3
\end{gathered}
$$

where $u, P, v$, and $\rho$ are the velocity, pressure, kinetic viscosity, and density, respectively. The SGS eddy viscosity $v_{S G S}$ is modeled following Smagorinsky as,

$$
v_{S G S}=\left(C_{s} f_{s} \Delta\right)^{2} \sqrt{2 \bar{S}_{i j} \bar{S}_{i j}}
$$

in which $\Delta$ is the volume of numerical elements and the model coefficient is given as Cs $=0.15$ in this study[7]. The following Van-Driest type damping function is adopted:

$$
f_{s}=1-\exp \frac{-y^{+}}{25}
$$




\section{Computational Conditions}

\section{Discretization}

The governing equations are discretized by the vertex-centered unstructured finite volume method. In the finite volume method, the governing equations are based on the following conservation equation in integral form.

$$
\frac{\partial}{\partial t} \int_{\Omega} \rho \phi \mathrm{d} \Omega+\int_{S} \rho \phi \boldsymbol{v} \cdot \boldsymbol{n} \mathrm{d} S=\int_{S} \Gamma \operatorname{grad} \phi \cdot \boldsymbol{n} \mathrm{d} S
$$

where the second on the left is the convective term and the right is the diffusion term. All dependent variables are defined on each vertex of numerical elements and a virtual control volume is constructed around the target vertex (see Fig. 2 in a simplified two dimensional case). Governing equations are integrated over the volume. The central finite difference scheme with the second order accuracy is basically applied to spatial discretization except for the convective fluxes which are treated by the MUSCL (Monotone Upwind Scheme for Conservation Law) scheme [8] to eliminate the excessive numerical oscillation. The diffusive fluxes on the volume surface are treated based on the deferred correction formula suggested by Muzaferija [9] to avoid the checker-board type oscillation.

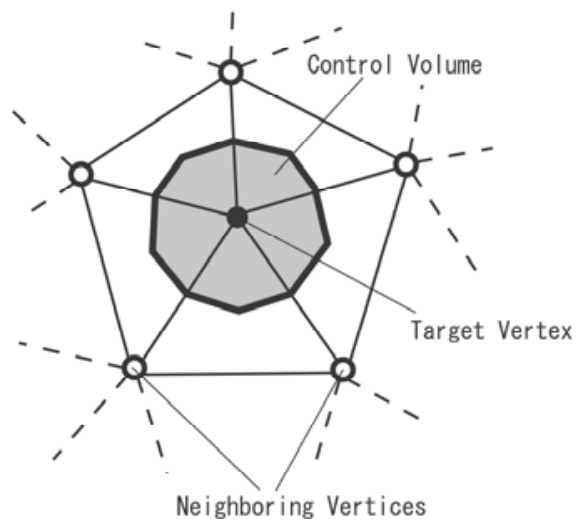

Fig. 2. Vertex-Centered Control Volume.

The 2nd order Adams-Bashforth scheme is adopted for time marching based on the SMAC (Simplified Marker and Cell) method proposed by Amsden and Harlow [10]. When constructing the pressure Poisson equation for the SMAC method, flow rate on the surface of the control volume is obtained following the method proposed by Rhie and Chow [11] to keep off the checker-board type pressure oscillation. Because the coefficient matrix of the discretized pressure Poisson equation is symmetric, the linear equation systems are solved by the ICCG (Incomplete Cholesky Conjugate Gradient) method.

The numerical dissipation included in the upwind scheme should preferably be avoided in the convective term wherever possible in LES. At the same time, in the engineering applications such as adopted here, the unstructured tetrahedral elements in the vicinity of the wall are seriously distorted and numerical oscillation is generated by the dispersive central finite difference. 


\section{Grid Resolution}

A total of about 38 million tetrahedron elements with 7 million numerical nodes were used to cover the entire computational domain. The surface resolution is restricted to be around $1 \mathrm{~cm}$ to capture the characteristic eddy structures found in the wind tunnel measurements. The spatial grids allocated around the vehicle, as well as the surface grid distributions, are shown in Fig. 3.

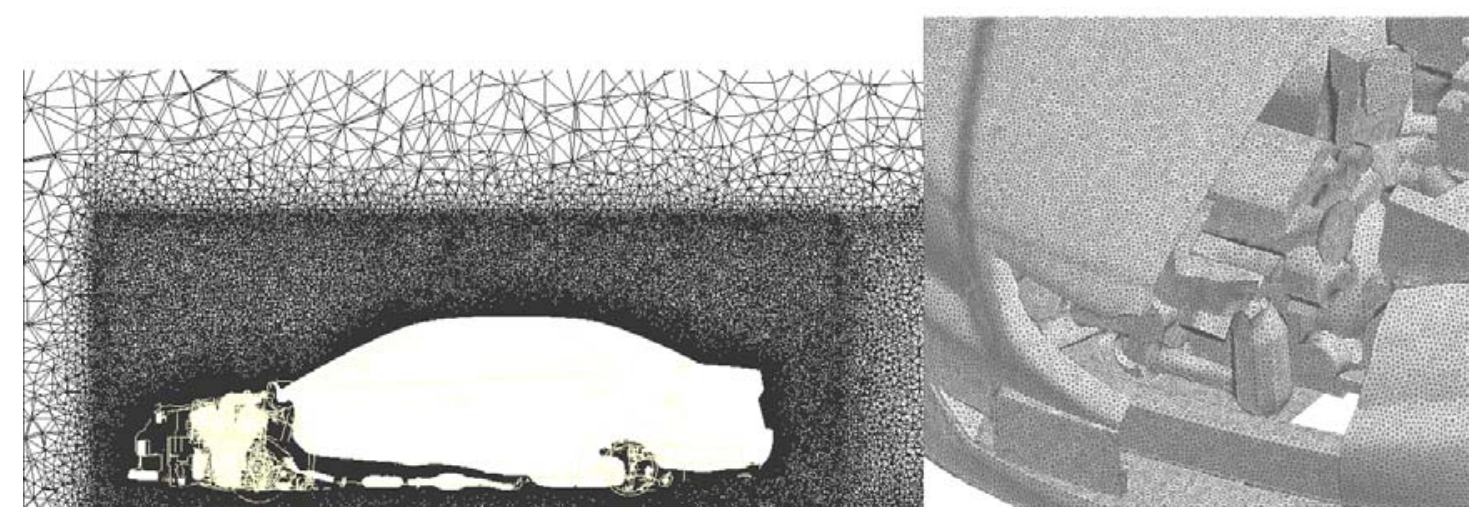

Fig. 3. Grid resolution around (left) and on the surface (right) of the vehicle.

\section{Boundary Conditions}

Boundary conditions for the computation are applied based on the wind tunnel test. Uniform velocity of $39 \mathrm{~m} / \mathrm{s}$ is imposed at the inlet, while all velocity components are gradient-free for streamwise direction at the outlet. For the accuracy of the outlet condition, we attached an additional layer consisting of prism elements at the exit of the domain to align the grid lines with the mean velocity direction.

On the surfaces of the car body, a solid wall condition is adopted. The typical wall distance of the first nearest gird point is measured from the numerical results and is less than 150 in wall unit $\left(y^{+}\right)$, which are located within the logarithmic layer of the mean velocity profile. The assumed log-law profile is directly applied to the instantaneous velocity field to estimate the surface friction. On the floor, free slip condition is applied in the region between the inlet and the front wheels to prevent the boundary layer from developing (shaded in Fig. 1). In the wind tunnel test, a boundary suction system is mounted on the floor in front of the car. The solid wall condition is imposed on the floor behind the car toward the outlet of the computational domain. The ceiling and the sidewall of the numerical domain are supposed to be free-slip on the surface.

\section{Hardware and Software}

The hardware on which we conducted the HPC-LES is the Earth Simulator (ES) in operation since 2002. The ES was developed under the national project by Japanese governmental agencies. The ES is a massive parallel vector supercomputer system of distributed memory and composed of 640 processor nodes (PN). Each PN consists of 8 vector-type CPU (peak performance is 8 Gflops/CPU) with a 16 Gbytes memory system, accordingly the total peak performance is 40 Tflops with 10 Tbytes memory. From 2002 to 2004, the ES has been recognized as the world's fastest supercomputer in the world, and still now, as a practical tool for engineering problems, the ES is one of the fastest in the world.

The computational code adopted is the FrontFlow/Red, which has been developed under the project of "Frontier Simulation Software for Industrial Science (FSIS)" organized at the University of Tokyo. The project started in 2002 as an IT-program research project sponsored by Ministry of Education, Culture, 
Sport, Science and Technology (MEXT). The code was optimized on the ES in this study under the succeeding renovated IT project "Revolutionary Simulation Software (RSS21)". High vectorization of $96 \%$ and parallelization of $99 \%$ have been achieved in the simulations on 100 PN and 800 CPU. With the performance having been achieved we could conduct one of the world's largest unsteady turbulence simulation of the flow around a formula car using 120 million meshes and more than 500 GB memory (e.g., Tsubokura et al. [12]).

In this study, we use 512 arithmetic nodes (CPU) on the ES. It takes about one and a half days for the calculation to reach the statistically steady state and to collect the reliable turbulence statistics. Total amount of computational memory consumed is about 300GB.

\section{Experimental Measurements}

Total pressure distributions around the full-size vehicle were measured in the Göttingen-type wind-tunnel at Mazda Motor Corporation. The test section is $12 \mathrm{~m}$ long and the section size is $6 \mathrm{~m}$ wide by $4 \mathrm{~m}$ height. Total pressure was measured by a keel tube with a diameter of $10 \mathrm{~mm}$, and a total of 90 tubes were mounted in a reticular pattern on a jig to measure the spatial pattern of the pressure distribution.

\section{RESULTS AND DISCUSSION}

\section{Comparison between LES and RANS on ASMO Model}

The numerical method adopted is quantitatively validated on the ASMO (Aerodynamisches Studien Modell) vehicle model [13]. The 1/5 wind tunnel test model (the length, the width, and the height are 0.81 $\mathrm{m}, 0.29 \mathrm{~m}$, and $0.27 \mathrm{~m}$, respectively) is adopted. The wind tunnel data were measured and provided by VOLVO Cars. Multiple grid configurations of 24.3 and 5.5 million tetrahedral elements are adopted to investigate accuracy and convergence of the numerical solutions. Grid resolutions on the surface of the vehicle are about 4 to $5 \mathrm{~mm}$, and 7 to $8 \mathrm{~mm}$ on 24.3, and 5.5 million elements, respectively. The overview of ASMO model and the computational domain are shown in Fig. 4. Surface grid distribution in the case of 24.3 million elements is illustrated.

The inlet mean velocity is set to $50.0 \mathrm{~m} / \mathrm{s}$ without turbulent intensity. Velocity boundary conditions are same as the ones for the full scale vehicle shown in Fig. 1. In addition to LES, we also conduct RANS simulation using the standard k epsilon model [14] to look into the validity of LES for the assessment of vehicle aerodynamics. For this purpose, we use exactly the same grid resolution for both LES and RANS. In RANS simulation, the convective term is discretized by the third order TVD scheme and time marching is done based on the first-order implicit Euler method. No special calibration is done on the model parameters. In LES, the time increment is set to $2.0 \times 10^{-6}$ sec and turbulence statistics are obtained for 90,000 time steps after the flow field reaches the fully developed turbulence state. In RANS, the convergence criterion for each variable is set to $10^{-4}$. Roughly speaking, LES is about one order of magnitude expensive compared with RANS in the context of CPU time to obtain the reliable turbulence statistics. 

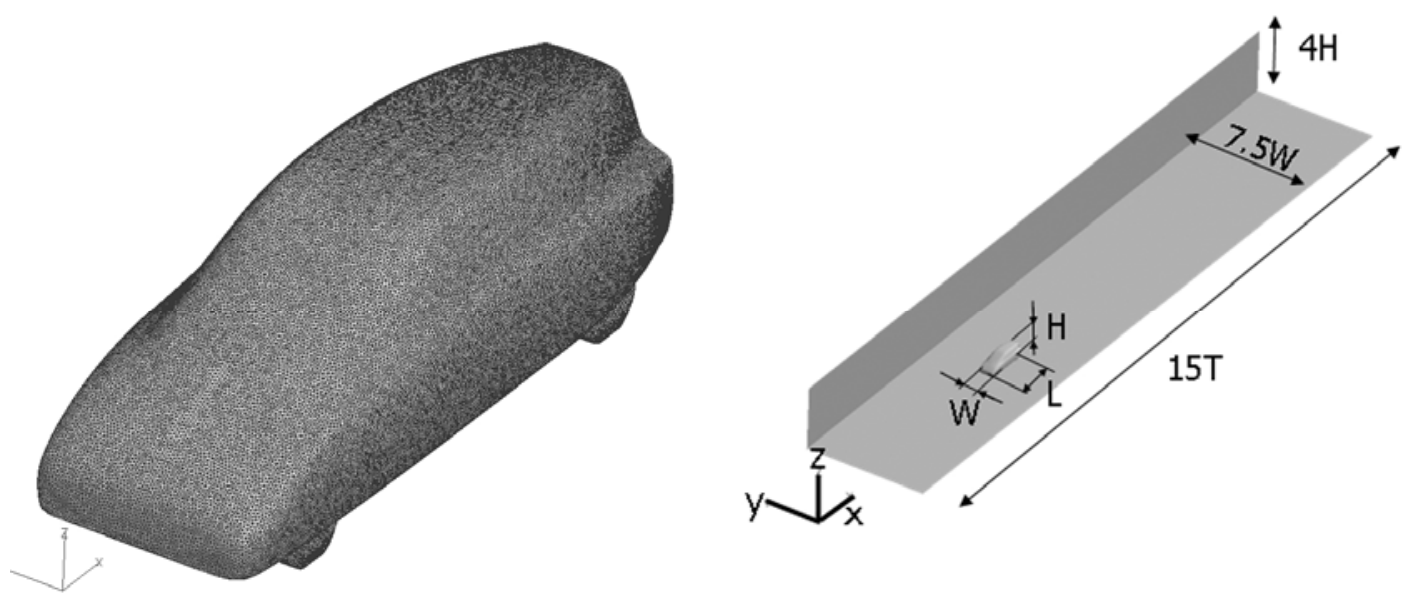

Fig. 4. Overview of ASMO model (left) and the computational domainon (right).

The time-averaged pressure distributions normalized by the inlet pressure and velocity along the centerline on the top and bottom surfaces of ASMO model are plotted in Fig. 5. Both LES and RANS show good agreement with the wind tunnel data on the roof side, while some discrepancies appear on the underbody side. The underestimation of RANS at the front region $(0.1<\mathrm{X} / \mathrm{L}<0.25)$ and the miss-prediction of the pressure drop at $\mathrm{X} / \mathrm{L}=0.8$ (the edge of the rear diffuser on the underbody) are remarkable, while LES clearly improves the problems of RANS. The mean pressure distributions on the rear end surface of the vehicle are illustrated in Fig. 6 . The base pressure significantly contributes to the total drag of the vehicle, and hence good prediction is indispensable for the aerodynamic assessment tool. LES shows good agreement with experimental data, on the other hand RANS underestimates the base pressure. The difference of RANS between 24.3 and 5.5 million numerical elements is not significant and convergence of numerical solution is identified. This result suggests that discrepancy between the experimental plots and RANS results originates from the modeling error and cannot be eliminated without improvement or tuning of the turbulence model.

Problems of LES identified in Figs. 5 and 6 also should be mentioned. At the simplified shape of ASMO, convergence of LES results is also found between 24.3 and 5.5 million elements, however numerical oscillation is identified on the surface and the rear end of the roof. The oscillating distribution by LES may be caused by the insufficient grid resolution for the boundary layer, which is typically the case when we use the tetrahedral mesh in the vicinity of the solid wall. However, we can say that its influence is only restrictive near the solid surface and does not cause significant distortion on the prediction of the mean pressure distribution. 


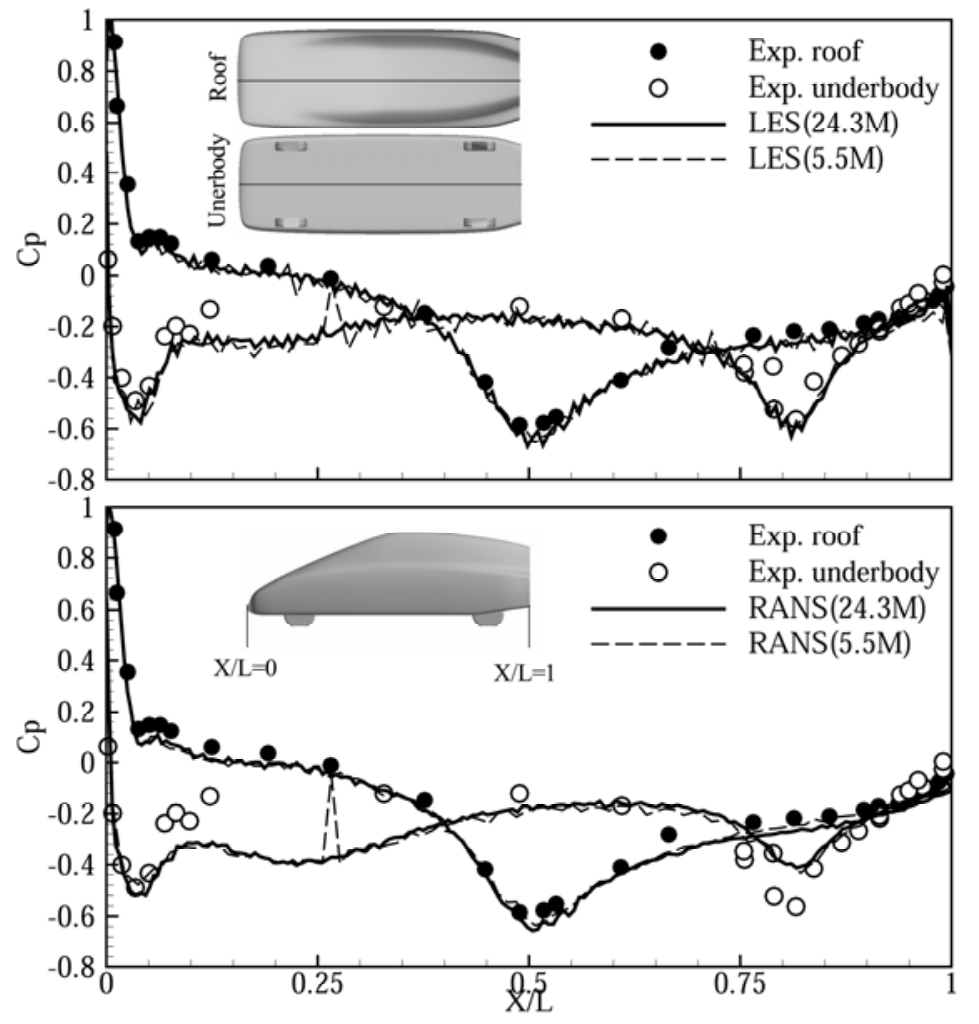

Fig. 5. Mean pressure distribution on the surface of ASMO by LES (above) and RANS (below).
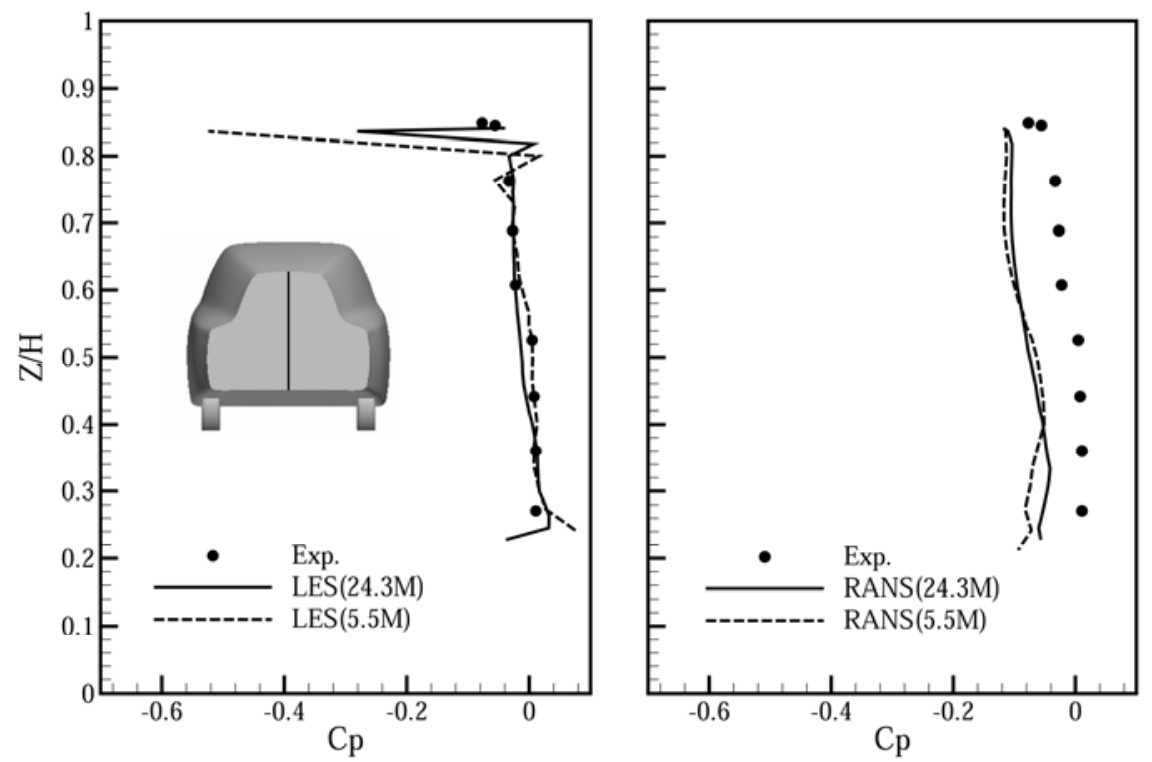

Fig. 6. Mean base pressure distribution of ASMO by LES (left) and RANS (right). 


\section{Vortex Structures around the Vehicle}

Unsteady flow structures on XY-, XZ-, and YZ-sections around the vehicle obtained from our numerical simulation are visualized in Fig. 7. The snapshots reveal that the flow on the upper side and front of the vehicle is relatively steady, while the wake formed after the surface flow detached from the roof trailing edge is strongly perturbed and unsteady. Structures of the wake is a dominant factor to determine the total aerodynamic performance of the vehicle, and is strongly affected by the four flows: the one detached from the vehicle roof, the one coming through the underbody, and the ones from the sides of the vehicle. In the previous study [15], they pointed out that underbody flow and thus the geometry of the underbody strongly affects the wake structures of vehicles. In fact, we can see that the underbody flow is perturbed by the underbody geometry as shown in Fig. 1 and interacts with the wake. Another important thing to be noted is that the flow coming through the engine room mingles with the underbody flow. Thus, inclusion of the complicated engine room in the numerical simulation for vehicle aerodynamics is indispensable even though it increases the cost for the simulation. We can also observe vortex-like structures on the sides of the vehicle, especially in the backward of the front tires, which are identified as blue round spots in the figures. The side vortices are formed by the front pillars, the door mirrors, and the front tire house. Vortex-like structures are also found in the underbody area.

The coherent vortices around the vehicle are more clearly visualized through the time-averaged pressure distributions. Figure 8 shows the iso-surfaces of the mean static pressure normalized by the inlet dynamic pressure. The most famous and dominant wake structure of the notchback-type vehicle is the pair of trailing vortex emanating from the rear pillars (e.g., Nouzawa et al.[16]). It is interesting to note that other vortices generated by the front pillars, the door mirrors, the front tire house, and possibly the underbody vortices mingle together at the rear end of the vehicle and contribute to form the trailing vortices. This fact suggests that all of these vortices around the vehicle more or less affect the near-wake flow structures and accordingly the total aerodynamic performance. 


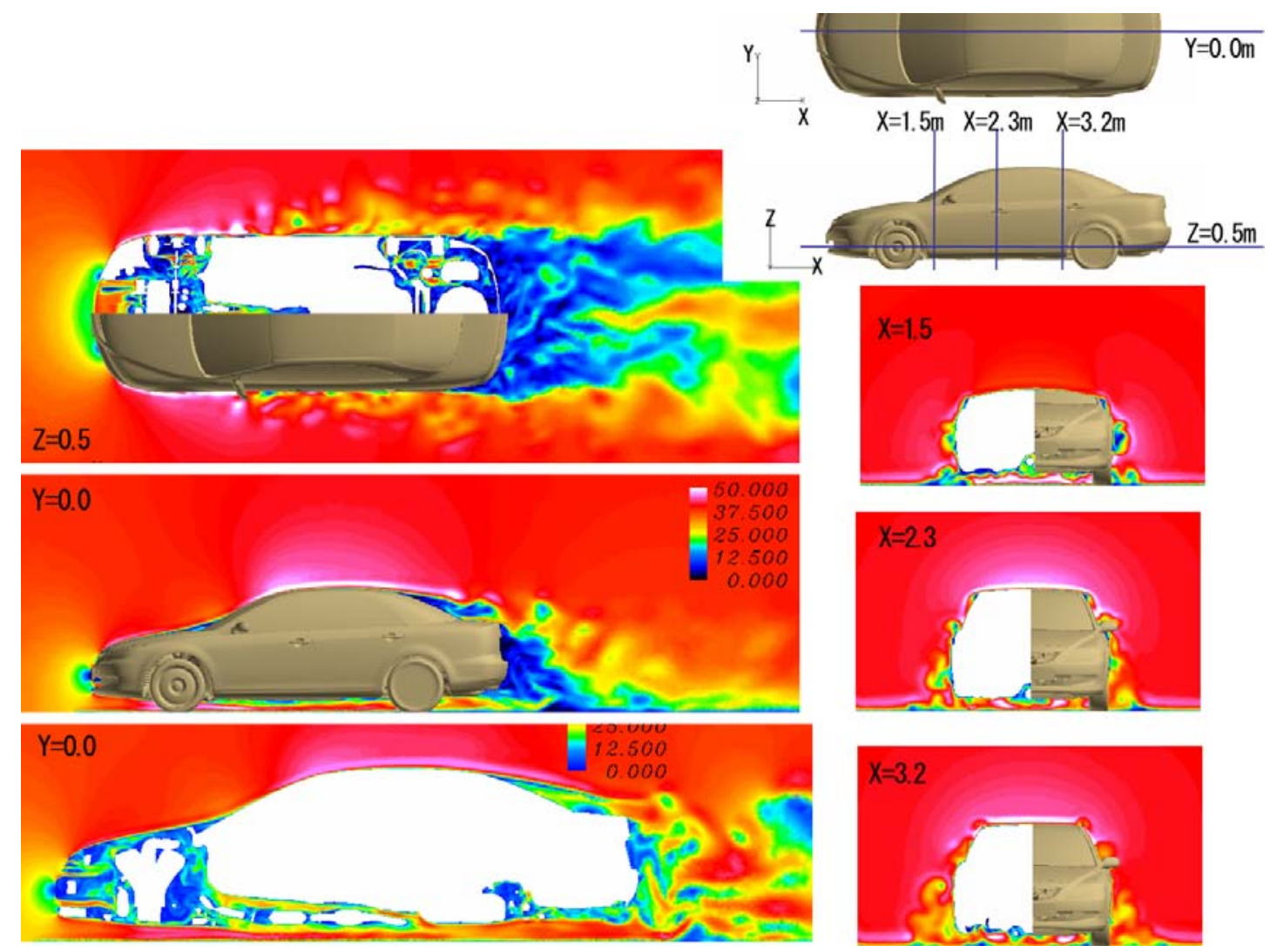

Fig. 7. Snapshots of the velocity magnitude around the vehicle.

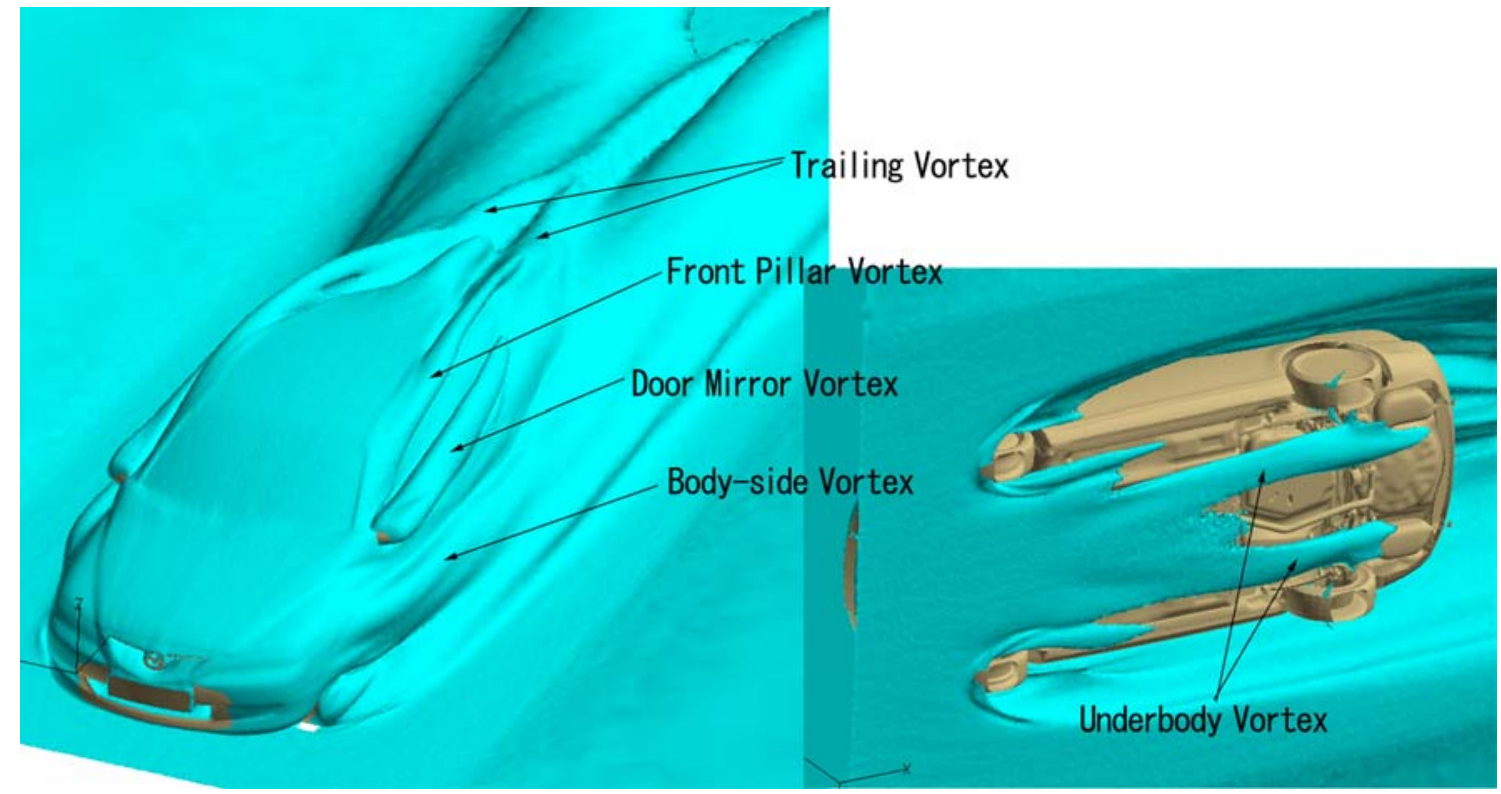

Fig. 8. Vortex structures around the vehicle (Iso-surface of the mean static pressure $\mathrm{Cp}=0.7$. 


\section{Validation with Experimental Data}

Flow structures reproduced by our unsteady simulation are validated using the full-scale experimental data measured in the wind tunnel. All figures in this section show the total pressure distributions. Figure 9 shows the wake structures visualized on the XZ-section at $\mathrm{Y}=0 \mathrm{~m}$ (central section). We can observe in the time-averaged numerical result (lower-right) that the wake is characterized by two flows: the one separated at the roof trailing edge with possible reattachment on the boot deck, and the one separated from the underbody trailing edge. As indicated in the experimental result (lower-left), our LES properly captures the so-called 'deadwater' region. The wake in the instantaneous field (upper-right) is more distorted and unsteady vortex-like structures shedding at the rear end of the vehicle can be found. In the previous study [17], it was shown that LES is superior to RANS in the prediction of the pressure distribution especially at the rear part of the vehicle (base pressure). They suggested that the reason is the capability of LES to reproduce the unsteady eddy structures found in the wake region.

Figure 10 also indicates the wake structures on the YZ-section at $250 \mathrm{~mm}$ behind the vehicle. The most notable structure on this section is the two projections attached on the top of the wake, which is clearly found in the experimental visualization. As shown in Fig. 8, the structure originates from the vortex produced by the front pillar. Because the front pillar vortex is widely acknowledged as the primary source of aerodynamic noise (e.g., Li et al. [18]), we can assume that improving the aerodynamic noise by controlling the front pillar vortex also affects the wake structures of the vehicle. Our LES reproduced the two projections well, while the third bulge appears between the two projections. Experimental data show a little sign of the existence of the third bulge.
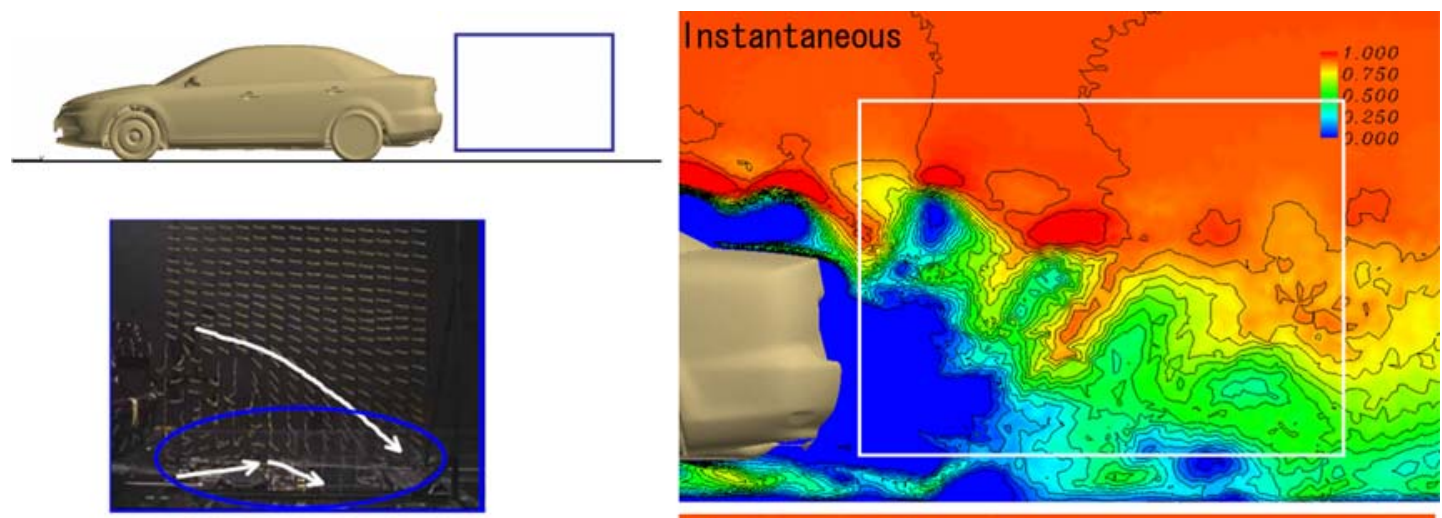

Experiments
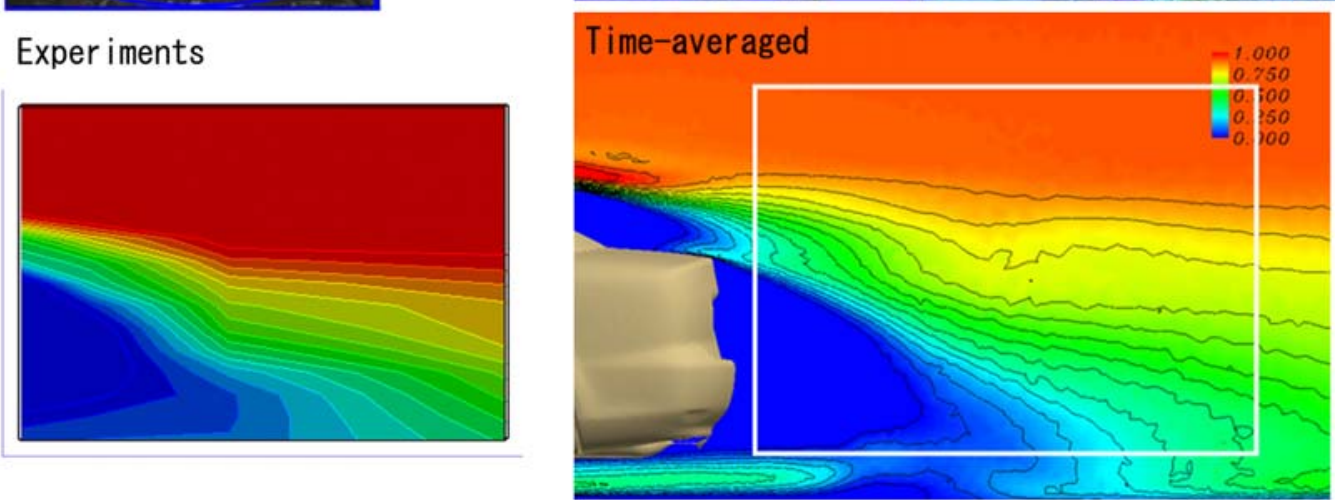

Fig. 9. Wake structures behind the vehicle on $X Z$-section at $Y=0 m$ (total pressure distributions): Left, experiments; Right, LES. 

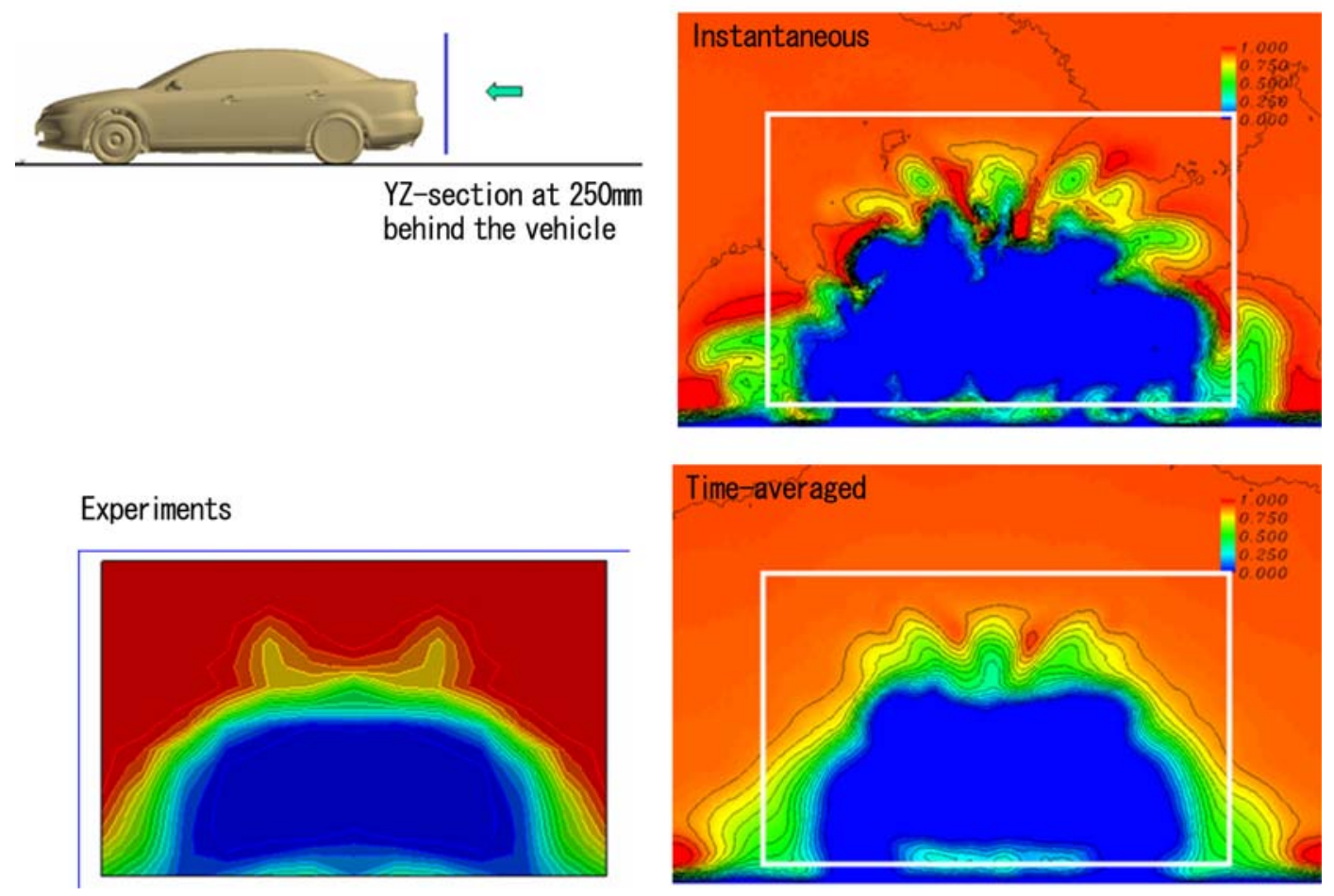

Fig. 10. Wake structures behind the vehicle on YZ-section (total pressure distributions): Left, experiments; Right, LES.

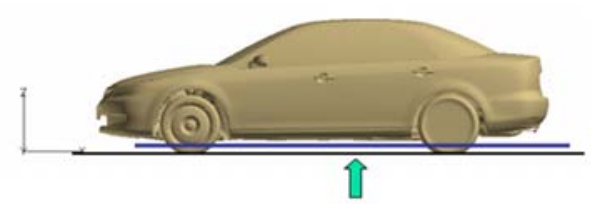

$\mathrm{XY}$-section at $100 \mathrm{~mm}$ above the ground

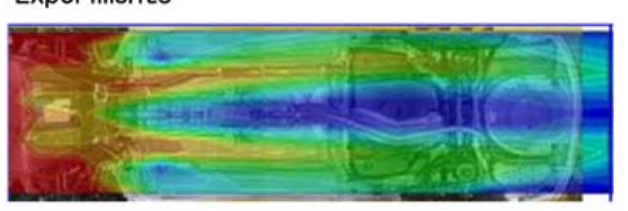

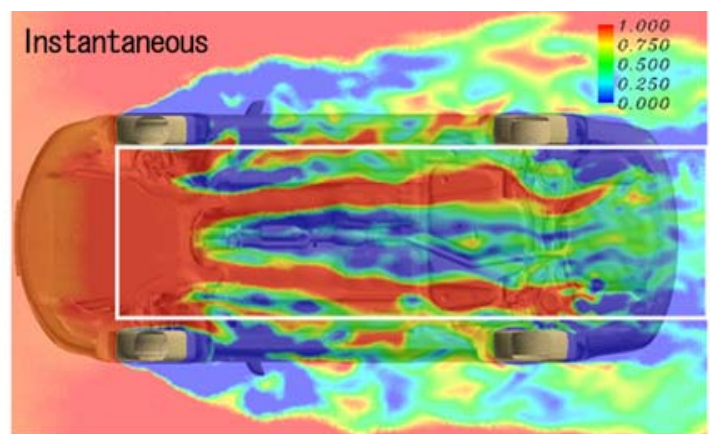

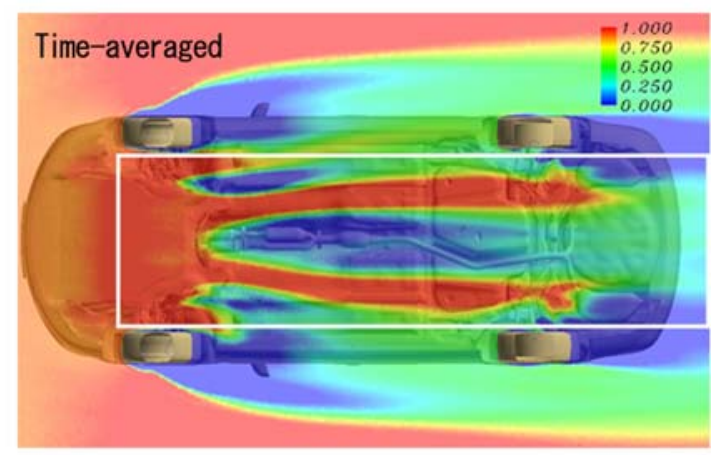

Fig. 11. Flow structures under the vehicle on $\mathrm{XY}$-section (total pressure distributions): Left, experiments; Right, LES. 
Flow structures under the vehicle on XY-section at $100 \mathrm{~mm}$ above the ground are visualized in Fig. 11. The flow coming directly through the front edge of the body interacts with the flow coming through the engine room. The wake of front or rear tires also interact with each other and overall underbody structures are quite complicated. Agreement of LES with the experimental data is qualitatively satisfactory, however the lower total-pressure region appearing laterally in the center of the figure is slightly wider estimated and the higher total-pressure regions elongated for the streamwise direction are overestimated by CFD. Further study must be done to determine the reason for the discrepancy; however, one possible explanation is the incomplete modeling for the engine room. In our CFD, a radiator mounted at the inlet of the real engine room was removed to avoid the excessive complicated geometry, which enhances the total inlet flow rate and thus the outlet flow from the engine room to the car underbody is overestimated.

Figure 12 illustrates flow structures on the side of the vehicle. The tires and tire houses, the side mirror, and the front pillar are the main source to disturb the side flow. Flow structure on the YZ-section at 300 $\mathrm{mm}$ behind the door mirror is shown at the top right. Two low pressure regions formed by the front pillar and the door mirror are identified in the experimental data and LES properly reproduces such structures. Other wake structures are found behind the tire houses, which are visualized at the bottom. Two low pressure regions produced by the front tire and its tire house are recognized as shown at the bottom left. These regions mingle together as the flow go downstream and form a large side wake (see the bottom right). LES moderately captures these flow features, but the side wake behind the rear tire house is estimated slightly wider. It is interesting to note that the wakes behind the door mirror and the front pillar flow a little upward and the latter attaches on the side edge of the roof, connecting the trailing vortices in the near-wake, which is more clearly observed in Fig. 8.
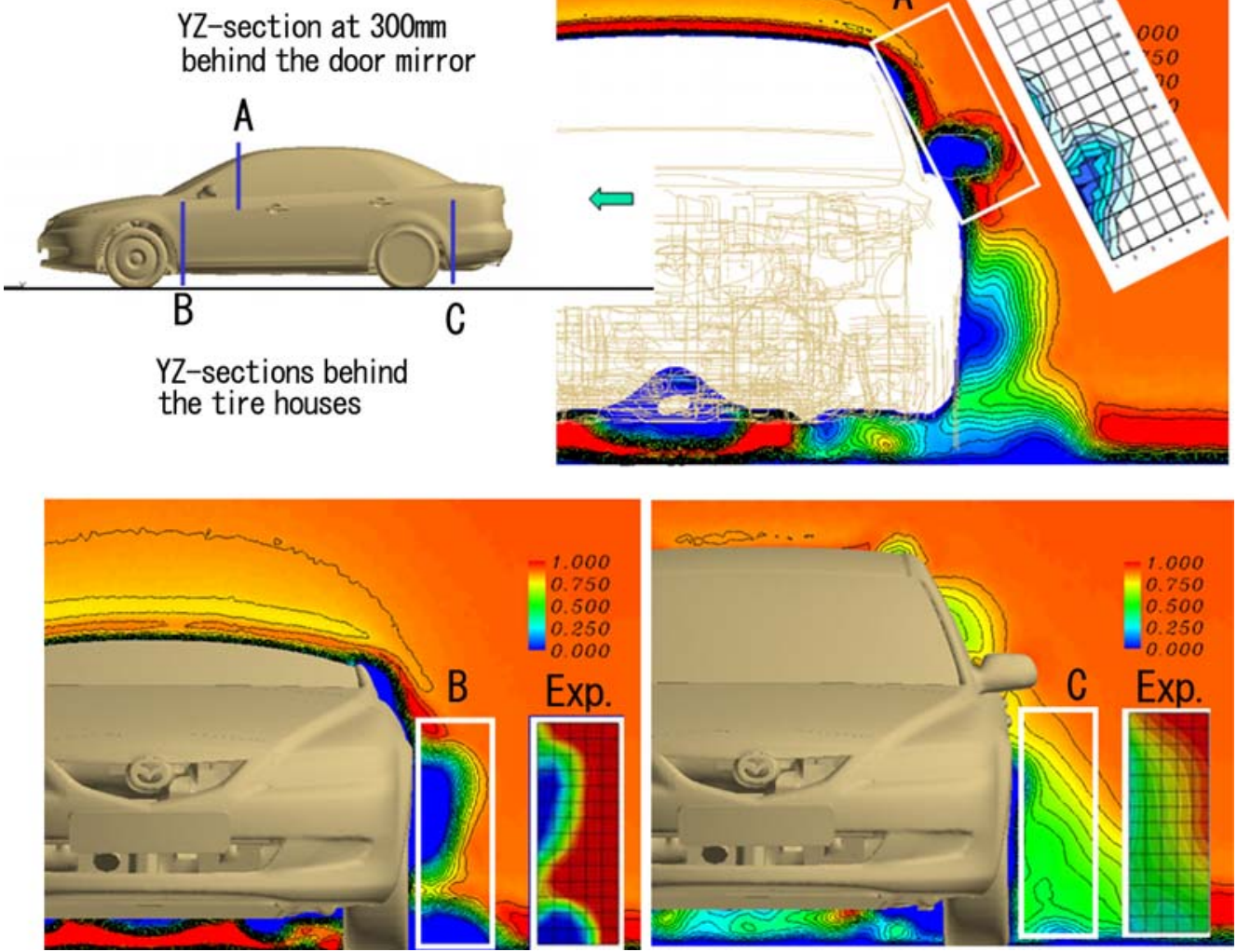

Fig. 12. Flow structures on the side of the vehicle (total pressure distributions). 


\section{Unsteady Flow Structures during the Dynamic Yaw-Angle Change}

A transient flow around the vehicle in the dynamic yaw-angle change is visualized to investigate the relationship between the flow and the unsteady aerodynamic forces. To mimic the sudden cross wind, the yaw angle of the vehicle against the main flow is changed from 0 to -10 degrees in 0.05 sec after the flow reaches a fully developed state, then the angle is fixed to -10 degree for $0.05 \mathrm{sec}$ and it is recovered to 0 degrees again in $0.05 \mathrm{sec}$, as illustrated in Fig. 13. We used the sliding grid method for the dynamic yaw-angle change.

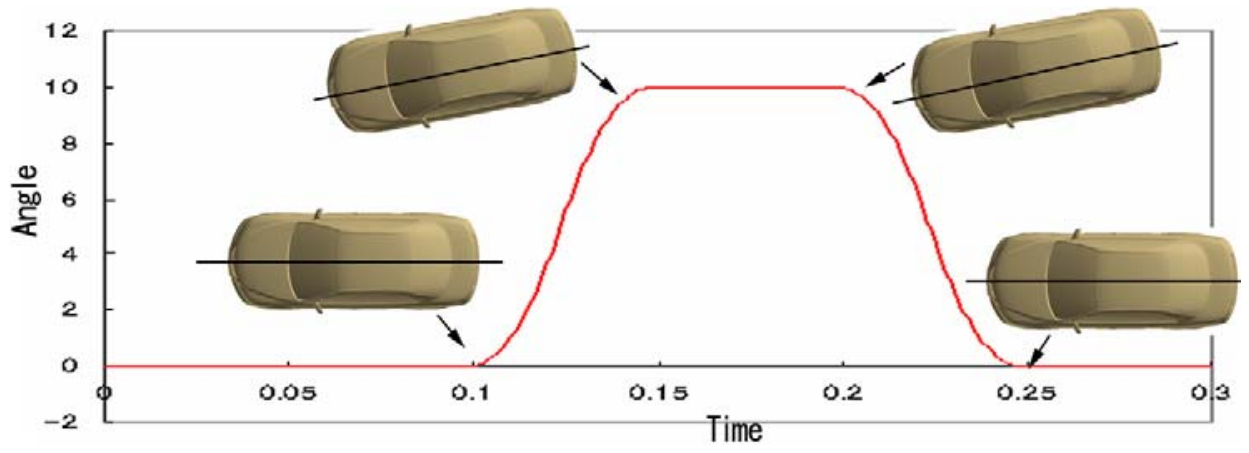

Fig. 13. Conditions for the dynamic yaw-angle change.

The pressure distributions normalized by the inlet dynamic pressure around the vehicle are shown in Fig. 14. Strong negative pressure regions can be observed at the front left and the rear right sides of the body at the end of the angle change from 0 to -10 degrees (XY-section at $\mathrm{T}=0.15 \mathrm{sec}$ ). Such asymmetric distributions on the left and right sides of the body is mitigated (but not perfectly by necessity) at $\mathrm{T}=0.2$ sec, which is $0.05 \mathrm{sec}$ after the angle is fixed to -10 degrees. The strong asymmetry of the pressure again appears when the yaw angle is recovered to 0 degree at $0.25 \mathrm{sec}$. This result clearly indicates the fact that, compared with the situation when the yaw angle is stationary, a relatively strong yawing moment acts on the car during the dynamic yaw-angle change. The figure also explains that, comparing those at $\mathrm{T}=0.15$ sec and $0.2 \mathrm{sec}$, the main reason of the unsteady yawing moment is the negative pressure on the rear of the body. Such an asymmetric pressure distribution is also identified on the YZ-section, on which pressure distribution on the left-half (in the figure) of the roof is slightly smaller at $\mathrm{T}=0.15 \mathrm{sec}$ than at $\mathrm{T}=0.2 \mathrm{sec}$. In the same way, the asymmetric pressure distribution on the roof is slightly stronger at $\mathrm{T}=0.25 \mathrm{sec}$ than $\mathrm{T}=0.2 \mathrm{sec}$.

The unsteady effects on the aerodynamic forces are clearly observed in the yawing and rolling moments. The time series of the yawing and pitching moments acting on the vehicle in the dynamic yaw-angle change are plotted in Fig. 15. The profiles overshoot during the dynamic yaw-angle change and the absolute value is about three or four times larger than the ones when the motion is static ( $\mathrm{T}=0.15$ $\sim 0.20 \mathrm{sec}$ ).

Finally the numerical problem found in the time series of the moments should be mentioned. The drastic jagged profiles of moments are caused by the violation of the mass conservation, which occurs as pressure oscillation in the process of interpolation on the connecting surface of the sliding mesh. The serious overshoot of the profile is typically eliminated within three or four time steps and velocity fields are not significantly affected by the pressure oscillation. 

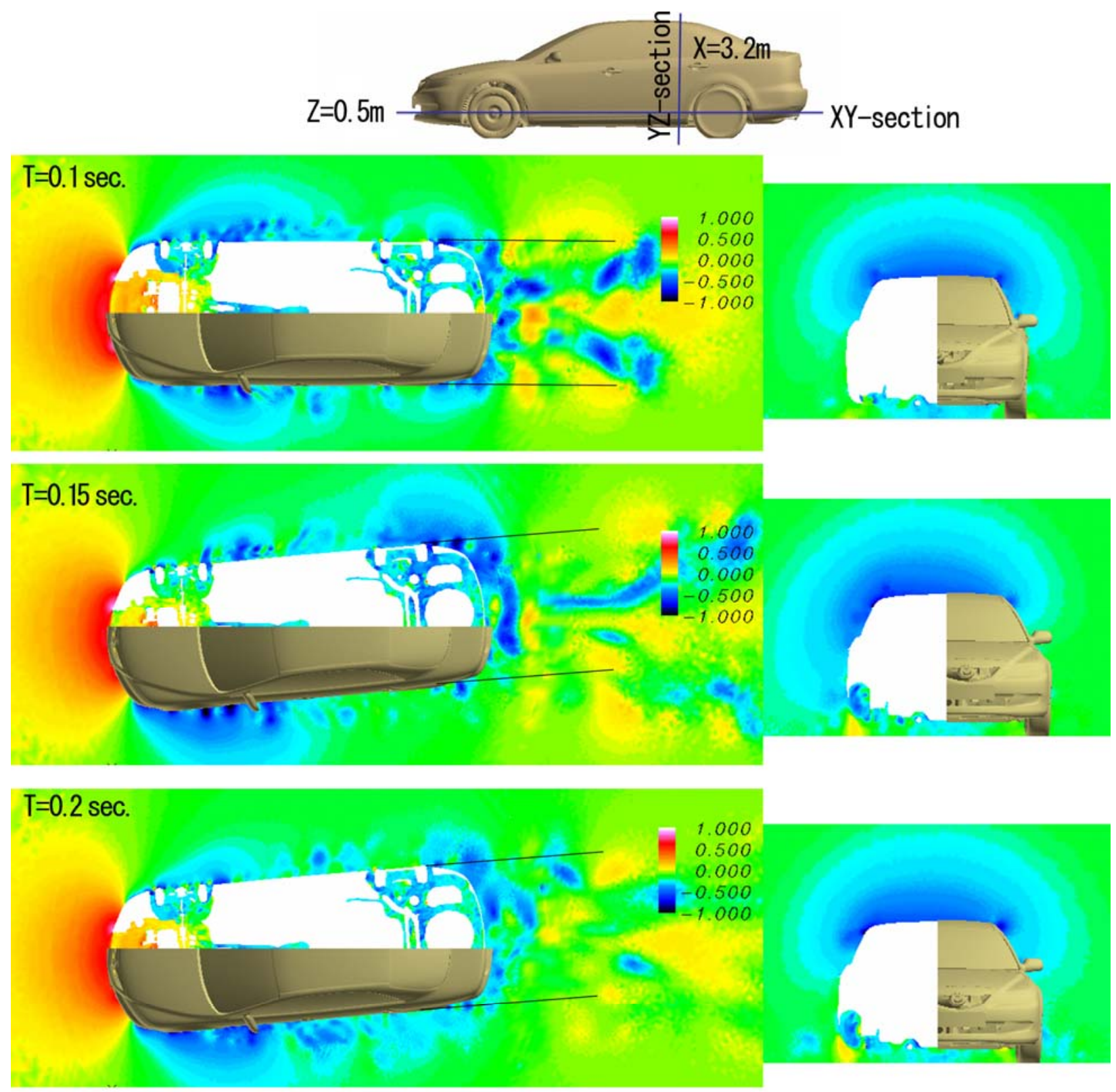

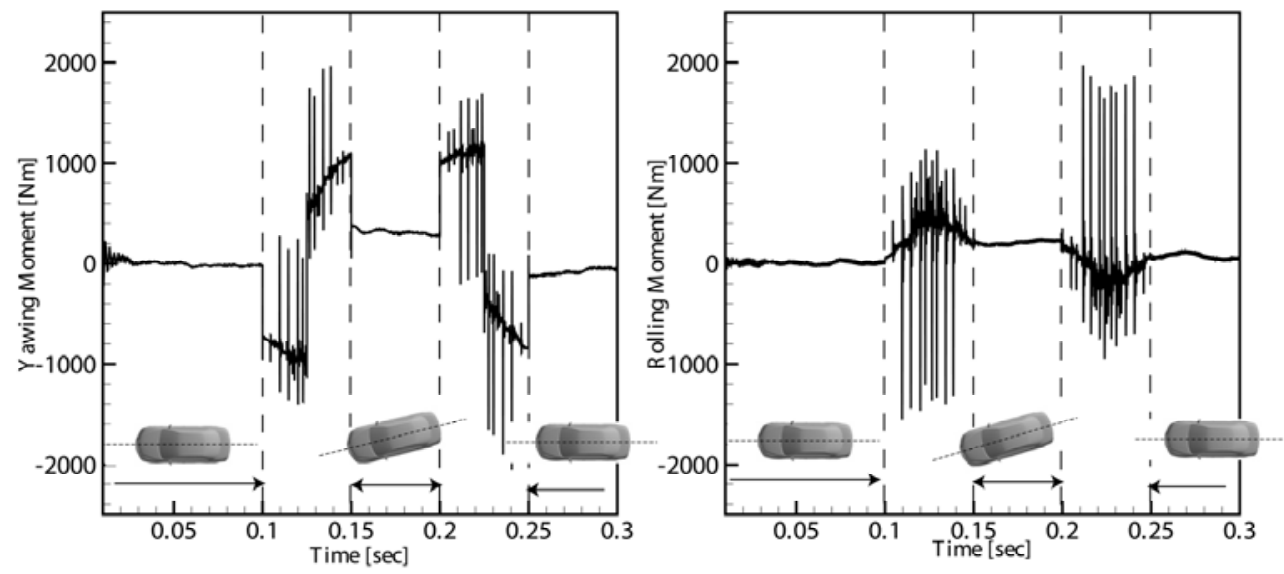

Fig. 15. Time series of the yawing (left) and pitching (right) moments.

\section{CONCLUDING REMARKS}

Unsteady flow structures around the production sedan were visualized using unsteady turbulence simulation (LES) and reproduced flow fields were validated with the experimental visualization obtained in the wind-tunnel test. Generally, the structures identified by the experiments were properly captured by our numerical simulation. Recently, in addition to the mean aerodynamic forces such as drag and lift coefficients, more attention is going to be paid to their unsteady components. One of the typical problems is the reduction of the aerodynamic noise generated around the front pillar. We have to identify the unsteady flow or eddy structures around vehicles and understand the physical mechanism of their generation and interaction with the solid body to solve such a problem. We have seen that various eddy structures around the vehicle are strongly interact with each other and relate to the twin trailing vortices, which determine the overall aerodynamic characteristics of the vehicle. As a result, it is demonstrated that LES will be a powerful tool for the vehicle aerodynamic assessment in the foreseeable future, because it can provide precious aerodynamic data which conventional wind tunnel tests or RANS simulations are difficult to provide.

\section{ACKNOWLEDGMENTS}

This work has been supported by the projects of FSIS and "Revolutionary Simulation Software (RSS21)" sponsored by MEXT, Japan. The CPU time provided by the collaboration project of the Earth Simulator Center is greatly appreciated.

\section{REFERENCES}

[1]Cogotti A. Generation of a Controlled Level of Turbulence in the Pininfarina Wind Tunnel for the Measurement of Unsteady Aerodynamics and Aeroacoustics. SAE Paper 2003; No. 2003-01-0430.

[2]Mayer J., Schrefl M., Demuth R. On Various Aspects of the Unsteady Aerodynamic Effects on Cars Under Crosswind Conditions. SAE Paper 2007, No. 2007-01-1548.

[3]Bearman PW, Mullarkey SP. Aerodynamic Forces on Road Vehicles due to Steady Winds and Gusts. Proceedings of meeting on Vehicle Aerodynamics held by Royal Aeronautical Soc. London 1994; 4.1-4.12.

[4]Yoshida Y., Muto S., Imaizumi T. Transient Aerodynamic Forces and Moments on Model of Vehicles Passing Through Cross-Wind. SAE Paper 1977, No.770391.

[5]Charuvisit S., Kimura K., Fujino, Y. Experimental and Semi-Analytical Studies on the Aerodynamic 
Forces Acting on a Vehicle Passing Through the Wake of a Bridge Tower in Cross Wind. Journal of Wind Engineering and Industrial Aerodynamics 2004; 92: 749-80.

[6]Coleman SA., Baker CJ. High Sided Road Vehicles in Cross Winds. Journal of Wind Engineering and Industrial Aerodynamics 1990; 36: 1383-92

[7]Deardorff JW. A Numerical Study of Three-Dimensional Turbulent Channel Flow at Large Reynolds Numbers. Journal of Fluid Mechanics 1970; 41: 453-80.

[8]van Leer B. Toward the Ultimate Conservative Difference Scheme. 4, A New Approach to Numerical Convection. Journal of Computational Physics 1977; 23: 276-99.

[9]Muzaferija S. Adaptive Finite Volume Method for Flow Predictions Using Unstructured Meshes and Multigrid Approach. PhD Thesis, University of London, 1994.

[10]Amsden AA., Harlow FH. A Simplified MAC Technique for Incompressible Fluid Flow Calculations. Journal of Computational Physics 1970; 6: 322-25

[11]Rhie C. M., Chow WL. A Numerical Study of the Turbulent Flow Past an Isolated Airfoil with Trailing Edge Separation. AIAA Journal 1983; 21: 1525-32.

[12]Tsubokura M., Nakashima T., Oshima N., Kitoh K., Zhang H., Onishi K., Kobayashi T.Large Eddy Simulation of Unsteady Flow Around a Formula Car on Earth Simulator. SAE Paper 2007; No. 2007-01-0106.

[13]Aronson D., Brahim S. B., Perzon S. On the Underbody Flow of a Simplified Estate. SAE Paper 2000; No. 2000-01-0485.

[14]Launder BE., Spalding DB. The Numerical Computation of Turbulence Flow. Computer Methods in Applied Mechanics and Engineering 1974; 3: 260-89.

[15]Kitoh K., Chatani S., Oshima N., Nakashima T., Sebben S., Large Eddy Simulation on the Underbody Flow of the Vehicle with Semi-Complex Underbody Configuration. SAE Paper 2007: No. 2007-01-0103.

[16]Nouzawa T., Haruna S., Hiasa K., Nakamura T., Sato H. Analysis of Wake Pattern for Reducing Aerodynamic Drag of Notchback Model. SAE Paper 1990; No. 900318.

[17]Tsubokura M., Kitoh K., Oshima N., Zhang H., Onishi K., Tominaga T., Kobayashi T., and Sebben, S. High Performance LES on Earth Simulator: A Challenge for Vehicle Aerodynamics. Transactions of FISITA 2007; No. F2006M111T.

[18]Li Y., Kamioka T., Nouzawa T., Nakamura T., Okada Y., and Ichikawa, N. (2003). Evaluation of Aerodynamic Noise Generated in Production Vehicle Using Experiment and Numerical Simulation. SAE Paper (2003); Paper 2003-01-1314,. 


\section{FIGURE CAPTIONS}

Fig. 1. The analysis model (left) and the computational domain (right).

Fig. 2. Vertex-Centered Control Volume.

Fig. 3. Grid resolution around (left) and on the surface (right) of the vehicle.

Fig. 4. Overview of ASMO model (left) and the computational domain (right).

Fig. 5. Mean pressure distribution on the surface of ASMO by LES (above) and RANS (below).

Fig. 6. Mean base pressure distribution of ASMO by LES (left) and RANS (right).

Fig. 7. Snapshots of the velocity magnitude around the vehicle.

Fig. 8. Vortex structures around the vehicle (Iso-surface of the mean static pressure $\mathrm{Cp}=0.7$ ).

Fig. 9. Wake structures behind the vehicle on XZ-section at $\mathrm{Y}=0 \mathrm{~m}$ (total pressure distributions): Left, experiments; Right, LES.

Fig. 10. Wake structures behind the vehicle on YZ-section (total pressure distributions): Left, experiments; Right, LES.

Fig. 11. Flow structures under the vehicle on XY-section (total pressure distributions): Left, experiments; Right, LES.

Fig. 12. Flow structures on the side of the vehicle (total pressure distributions).

Fig. 13. Conditions for the dynamic yaw-angle change.

Fig. 14. Snapshots of static pressure (Cp) around the vehicle on the dynamic yaw-angle change: Left, XY-sections; Right, YZ-sections.

Fig. 15. Time series of the yawing (left) and pitching (right) moments. 